\title{
Developing Intuition: Neural Correlates of Cognitive-Skill Learning in Caudate Nucleus
}

\author{
Xiaohong Wan, ${ }^{1}$ Daisuke Takano, ${ }^{3}$ Takeshi Asamizuya, ${ }^{2}$ Chisato Suzuki, ${ }^{2}$ Kenichi Ueno, ${ }^{2}$ Kang Cheng, ${ }^{1,2}$ Takeshi Ito, ${ }^{3}$ \\ and Keiji Tanaka ${ }^{1}$ \\ ${ }^{1}$ Cognitive Brain Mapping Laboratory and ${ }^{2}$ Support Unit for Functional Magnetic Resonance Imaging, RIKEN Brain Science Institute, Wako, Saitama \\ 351-0198, Japan, and ' Department of Communication Engineering and Informatics, Graduate School of Informatics and Engineering, The University of \\ Electro-Communications, Chofu, Tokyo 182-8585, Japan
}

The superior capability of cognitive experts largely depends on automatic, quick information processing, which is often referred to as intuition. Intuition develops following extensive long-term training. There are many cognitive models on intuition development, but its neural basis is not known. Here we trained novices for 15 weeks to learn a simple board game and measured their brain activities in early and end phases of the training while they quickly generated the best next-move to a given board pattern. We found that the activation in the head of caudate nucleus developed over the course of training, in parallel to the development of the capability to quickly generate the best next-move, and the magnitude of the caudate activity was correlated with the subject's performance. In contrast, cortical activations, which already appeared in the early phase of training, did not further change. Thus, neural activation in the caudate head, but not those in cortical areas, tracked the development of capability to quickly generate the best next-move, indicating that circuitries including the caudate head may automate cognitive computations.

\section{Introduction}

There are cognitive experts, who have superior capability of problem solving in various domains, including board games, mathematics, medical diagnoses, and auditing. The cognitive expertise is domain specific, and acquiring cognitive expertise requires a long-term, deliberate practice in the particular domain (Ericsson and Lehmann, 1996). Similar to motor skill acquisition, novices perform the task with conscious effort at the initial phase of learning. As knowledge and experience accumulate, however, performance becomes smoother and can be executed with little conscious effort (Fitts and Posner, 1967; VanLehn, 1996). This unconscious capability of quick, automatic cognitive information processing is often referred to as intuition (De Groot, 1986; Klein, 2003; Gobet and Chassy, 2009). Psychological models have been proposed to account for the development of intuition (Simon and Chase, 1973; Anderson, 1982), but the validity of these models and their underlying neural basis are not yet established.

By comparing professional players with amateurs in the game of shogi (Japanese chess), we recently confirmed that quick generation of the best next-move occurred more reliably in professionals than in amateurs and then found that activation in the

Received May 14, 2012; revised Sept. 12, 2012; accepted 0ct. 5, 2012.

Author contributions: X.W., D.T., K.C., T.I., and K.T. designed research; X.W., D.T., T.A., C.S., K.U., and T.I. performed research;X.W. contributed unpublished reagents/analytic tools; X.W. and K.T. analyzed data; X.W., K.C., and K.T. wrote the paper.

This work was supported by the Fujitsu Laboratories.

This article is freely available online through the J Neurosci Open Choice option.

Correspondence should be addressed to Keiji Tanaka at the above address. E-mail: keij@ @riken.jp.

DOI:10.1523/JNEUROSCI.2312-12.2012

Copyright $\odot 2012$ the authors $\quad 0270-6474 / 12 / 3217492-10 \$ 15.00 / 0$ head of caudate nucleus, a dorsal part of the basal ganglia, but not cortical activation, was specifically associated with this process (Wan et al., 2011). The caudate head activation was specific in that it was less prominent in amateur players and was absent during deliberative search in either subject group. These findings associated the capability of quickly generating the best next-move to the caudate head. However, a critical unaddressed issue was whether different activation patterns in the caudate head between professionals and amateurs could be explained by their differential training histories, or by differences in intrinsic brain function that existed before the training (Poldrack, 2000). Therefore, it remains to be determined whether activation in the caudate head emerges in association with the development of quick generation capability through extensive training.

Although there have been a few imaging studies on the effects of cognitive practice on brain activities, most of them merely examined activity changes within a session limited to a day (e.g., Kelly and Garavan, 2005). The cognitive expertise, such as that of board game experts, requires training over a much longer period (Simon and Chase, 1973; Ericsson and Lehmann, 1996). Several imaging studies have also measured the changes over weeks of cognitive practice, but the tasks in these studies were mirror reading, spatial working memory, working memory updating, or sensory-motor mappings in dual tasks (Poldrack and Gabrieli, 2001; Olesen et al., 2004; Erickson et al., 2007; Dahlin et al., 2008; Dux et al., 2009). The relation of these tasks to the domain-specific cognitive expertise is not clear. Here, using a longitudinal (15 weeks) within-subject experimental design, we examined whether the activation in the caudate nucleus would emerge when novices acquired the capability to quickly generate the best next-move after exten- 
A

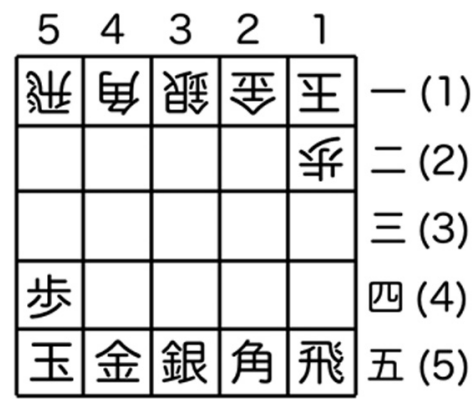

B
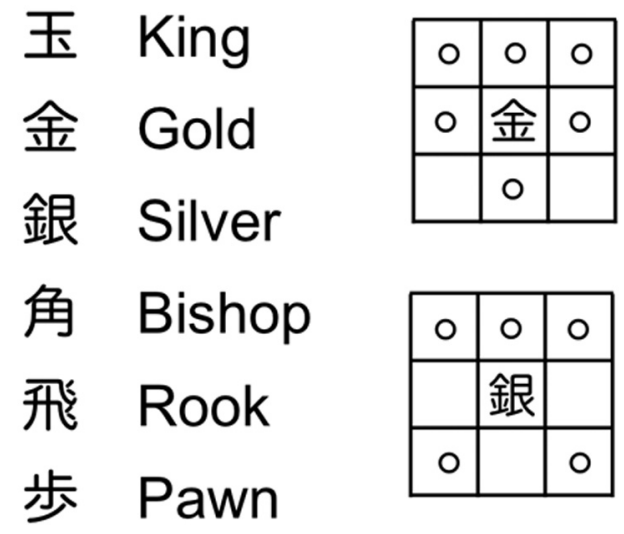

Figure 1. Overview of mini-shogi. A, Starting setup. The board is composed of 5 by 5 positions. The horizontal positions are expressed in Arabic numerals, and the vertical positions in Chinese numerals. B, Six types of pieces and their movability. King, Rook and Bishop have corresponding types in chess with identical movability, whereas Gold and Silver are unique to mini-shogi (and shogi). Pawn can take the opponent's piece at the position directly forward, but not oblique forward.

sive practice. We also assessed the possibility that the development of quick generation capability may be constrained by certain brain structures.

\section{Materials and Methods}

Subjects. Twenty Japanese male undergraduate students (20 22 years old, right-handed), who had little knowledge or experience in the games of shogi and mini-shogi (confirmed by some relevant questions and problems), participated in the project. Informed consents were obtained from each subject in accordance with a protocol approved by the ethics committees of the University of Electro-Communications (for training) and of RIKEN (for fMRI experiments). One of the subjects stopped regular practice in the middle of training. His data were not included in the main parts of analyses.

Characteristics of shogi compared with chess. Shogi is similar to chess in several aspects including the game objective, which is to take the opponent's King (checkmate). There are eight types of pieces in shogi. Many of the piece types have corresponding types in chess with identical movability, whereas some are unique to shogi, such as Gold, Silver, and Lance. In shogi, the Knight can only move to one of two forward positions, and the Pawn can take the opponent's piece at the position one square directly forward (oblique forward in chess). There is no analog of the chess Queen in shogi.

However, shogi has some unique rules different from chess. First, the opponent's pieces taken from the board can stay in the reserve and return into play as ally pieces at any time ("drop rule"). Because of this drop rule, the number of possible moves at a given board position is larger ( $\sim 80 \mathrm{vs}$ $\sim 40)$, and the average total number of moves in a game is larger $(\sim 140$ vs $\sim 80$, including moves by both sides) in shogi than those in chess. Second, most types of pieces can be promoted to be stronger (with wider movability) when they enter the bottom three rows in the opponent's side. The promotion provides Rook and Bishop with the

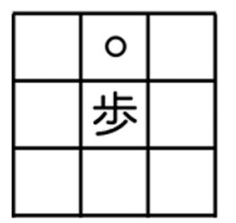

movability of King in addition to their original movability, and converts Silver and Pawn to Gold. When promoted pieces are captured by the opponent, they return to their original types.

Characteristics of mini-shogi compared with shogi. Mini-shogi is a simplified version of shogi. The board is smaller ( 5 by 5 in minishogi and 9 by 9 in shogi) and there are fewer types of pieces (Fig. 1). Consequently, games of mini-shogi are simpler than those of shogi. The number of possible moves at a given board position is smaller ( $\sim 20 \mathrm{vs} \sim 80)$, and the average total number of moves in a game is smaller ( $\sim 40$ vs $\sim 140$, including moves by both sides) in mini-shogi than those in shogi. The promotion can occur when the piece enters the bottom row in the opponent's side.

Training. The subjects played mini-shogi games daily for 15 weeks, mostly against a computer program named K55-shogi. The subjects chose the appropriate level of the opponent's skill in the computer program. They gradually raised the level of the computer opponent. In addition to the games against computer, the subjects also played games online or face-toface with other players. They were allowed to learn from shogi books and databases. The subjects weekly reported their total learning time and their levels in terms of the level of K55-shogi computer program against which they won $\sim 50 \%$ of games. They were asked to learn at least one hour per day, but the average learning time was actually $40 \mathrm{~min}$ per day.

Each subject received 20,000 yen each month. In addition to this base payment, to motivate the subjects to actively practice, awards were provided according to the results at a tournament held at the end of the long-term training. The subjects who were ranked as the top five received 100,000 yen, those ranked from the sixth to tenth received 50,000 yen, and the others received no awards. The rankings were determined by a Swiss style tournament with eight rounds.

$f M R I$ experiments. For the fairness of the tournament scheduled at the end of training, all subjects started to practice mini-shogi games on the same day. The first fMRI experiments (fMRI1) were carried out at the second or third week (12.7 \pm 4.1 days), and the second fMRI experiments (fMRI2) were carried out at the fourteenth or fifteenth week (99.7 \pm 3.2 days) of the training (Fig. $2 A)$. The high-resolution anatomical structure images were also taken in the two experiments.

The subjects viewed images for tasks on a rear projection screen through a mirror (resolution, $1024 \times 768$ pixels; refresh rate, $60 \mathrm{~Hz}$ ). Normal or corrected-to-normal vision was achieved for each subject. All images were restricted to a $<6$ degree square area surrounding the fixation point. The position of the left eye and its pupil size were monitored during the experiments.

In fMRI experiments, we measured the brain activity associated with the quick generation of the idea of the best next-move to a given board pattern (problem). The pattern mimicked an end phase of a mini-shogi game. The final checkmate (capturing the opponent's King) can be reached by one move in about a half of the problems, by three moves (including one move by the opponent) in a quarter, and by five moves in the remaining quarter. After $1 \mathrm{~s}$ of eye fixation on a fixation point, the board pattern of a mini-shogi problem was presented for $2 \mathrm{~s}$, and then the subject had to select one from the four options within $3 \mathrm{~s}$ by pressing one of four buttons (Fig. 2B). Each trial lasted for $11 \mathrm{~s}$, and in the remaining time the subject was engaged in a simple detection task: detecting a "Gold" piece from serially presented shogi pieces (each piece was presented for $150 \mathrm{~ms}$, followed by a mask for $100 \mathrm{~ms}$; thus, four pieces were presented per second). The subject reported the appearance of the 


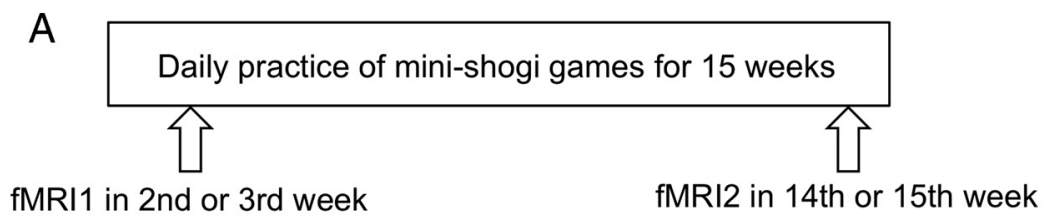

B

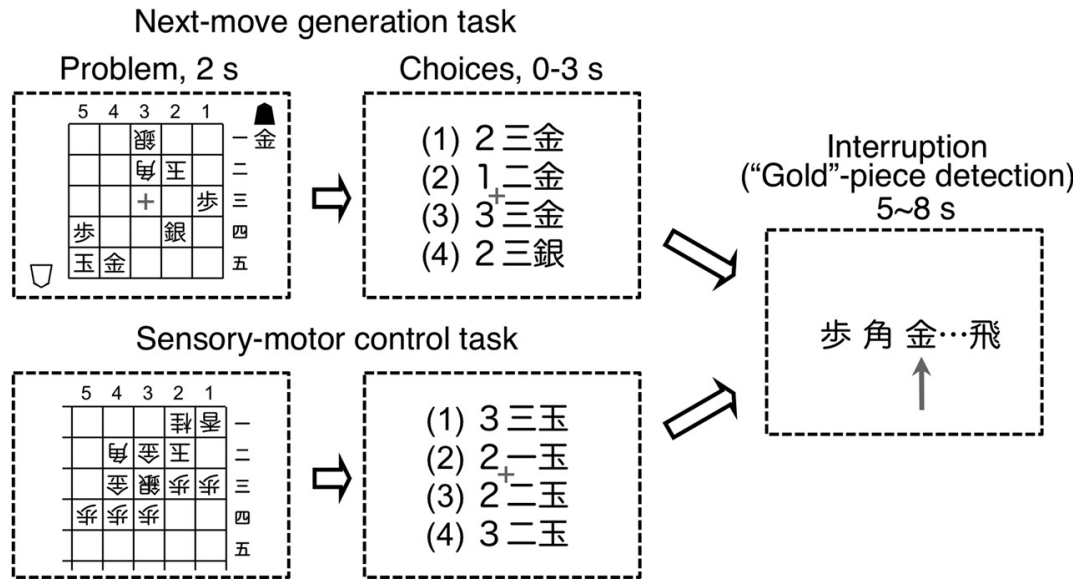

Figure 2. The overall experimental procedure and the tasks used in fMRl experiments. $A$, fMRI was conducted in the early ( $\sim 2$ weeks, $f M R I 1)$ and late ( $\sim 14$ weeks, fMRI2) phases of the 15 -week long training with mini-shogi games. $\boldsymbol{B}$, Trials of the task to quickly generate the best next-move were intermingled with those of the sensory-motor control task. In the quick generation task, a board pattern was presented briefly (for $2 \mathrm{~s}$ ) and then it was replaced with four possible next-moves. The subject was instructed to generate an idea of the best next-move that would reach a checkmate during the board-pattern presentation period and to select one of the four choices within 3 s according to their matches with the idea. The event sequence in the control task was similar, but the board pattern contained only the opponent's pieces. The subject selected one of the four choices that indicated the King's position. The next moves are indicated by the horizontal (first letter in Arabic numeral) and vertical (second letter in Chinese numeral) positions of the piece after the move and the type of the piece (third letter). The King's position was indicated in the same format in the control task. An interruption task in which the subject detected a "Gold" piece in sequentially presented pieces followed the main tasks to stop the subject's thinking of the problem.

"Gold" piece by pressing a button (Fig. 2B). In the sensory-motor control task, the sequence was the same but the subject reported the King's position in an opening shogi pattern composed only of the opponent's pieces (Fig. 2B). Because the pattern didn't contain the subject's own pieces, there was no way to think about the next move. One hundred and eighty trials of the best next-move generation task and 60 trials of the sensory-motor control task were randomly intermingled and given in four sessions. The subject was instructed to fix his gaze at the fixation point throughout the trials. The same problem for the task to quickly generate the best next-move or the same pattern for the sensory-motor control task never appeared again within an experiment. However, of the 180 mini-shogi problems used in fMRI1, 160 were used again in fMRI2 to assure the same problem difficulty in the two experiments. The order of their presentations was randomly determined. All patterns for the sensory-motor control task were also used repeatedly. The behavioral and fMRI data analyses were based on the 160 repeated problems.

Before fMRI1, each subject was trained for the task in five short sessions: three sessions outside the scanner, each composed of five problems, and two sessions inside the scanner, each composed of 15 problems. Before fMRI2, each subject practiced two sessions inside the scanner, each composed of 15 problems. The problems used in these practices were not used in the main experiments.

The durations of the problem presentation ( $2 \mathrm{~s}$ ) and response window (3s) were determined in preliminary experiments with four subjects who did not take part in the main experiment. They practiced mini-shogi games for 3 months and then participated in psychological experiments. We started with problem presentation of $1 \mathrm{~s}$ and response window of $2 \mathrm{~s}$ and gradually increased both durations until the subject's correct responses were significantly better than the chance.

MRI parameters. All MRI experiments were conducted using a 4 T MRI system with a head gradient coil (Agilent). Functional and highresolution anatomical images were acquired by parallel imaging with a bird-cage RF transmit coil and a 4 channel phased-array receive coil.

Functional images were acquired using the TSENSE technique (Kellman et al., 2001) with a two-segment, centric-ordered gradient echo $\mathrm{T}_{2}^{\star}$ echo-planar imaging (EPI) sequence with volume repetition time (TR) of $2 \mathrm{~s}$, echo time (TE) of $15 \mathrm{~ms}$, slice thickness of $4 \mathrm{~mm}$, and in-plane resolution of $3.0 \times 3.0 \mathrm{~mm}^{2}$ (field of view, $19.2 \times 19.2 \mathrm{~cm}^{2}$; flip angle, $40^{\circ}$ ). Thirty slices were collected with interleaved acquisition in an oblique orientation at $15^{\circ}$ titled from the anterior commissure-posterior commissure (AC-PC) line (which goes down anteriorly). In-plane 2D anatomical images were also collected with a four-segment $\mathrm{T}_{1}$-weighted MPRAGE (magnetization-prepared rapid gradient echo) sequence. Accompanying low-resolution 3D $\mathrm{T}_{1}$-weighted anatomical images (resolution, $1.72 \times 1.72 \times 1.72 \mathrm{~mm}^{3}$ ) in each experiment were also scanned in the same slice orientation as in the EPI acquisition.

Both $\mathrm{T}_{1}$ and $\mathrm{T}_{2}^{*}$-weighted anatomical images were collected using $3 \mathrm{D}$ MPRAGE sequence (inversion time, $500 \mathrm{~ms}$ for $\mathrm{T}_{1}$ images and without the inversion pulses for $\mathrm{T}_{2}^{*}$ images; $\mathrm{TR}=12.7 \mathrm{~ms}$; $\mathrm{TE}=6.8 \mathrm{~ms}$; resolution, $1.0 \times 1.0 \times 1.0 \mathrm{~mm}^{3}$ ).

$f M R I$ data analyses. Image processing and analyses were performed using BrainVoyager (Brain Innovation). To correct for the rigid head motion, all EPI images were realigned to the first volume of the first scan. Datasets in which translation motions were larger than $1.0 \mathrm{~mm}$ or rotation motions were larger than 1.0 degree were discarded. Functional EPI images were then registered, first to $2 \mathrm{D}$ anatomical images, then to lowresolution $3 \mathrm{D} \mathrm{T}_{1}$ anatomical images, and finally to high-resolution $3 \mathrm{D} \mathrm{T}_{1}$ anatomical images. They were spatially transformed into Talairach space (Talairach and Tournoux, 1988) by resampling the data with a resolution of $2 \times 2 \times 2 \mathrm{~mm}^{3}$. A spatial smoothing with a $4 \mathrm{~mm}$ Gaussian kernel (full width at half-maximum) and a high-pass temporal filtering with a cutoff of $0.005 \mathrm{~Hz}$ were applied to all fMRI data.

Statistical parametric maps were created using multiple regression analysis. Each trial was modeled with two regressors defined for: (1) the period of presentation of mini-shogi patterns, together with the response time difference (defined as the time obtained by subtracting the averaged response time that the subject spent in the sensory-motor control task from the response time) in each trial; and (2) the period of "Gold"-piece detection. The sum of the mini-shogi pattern presentation period and the subsequent response time difference was regarded as the time to generate 

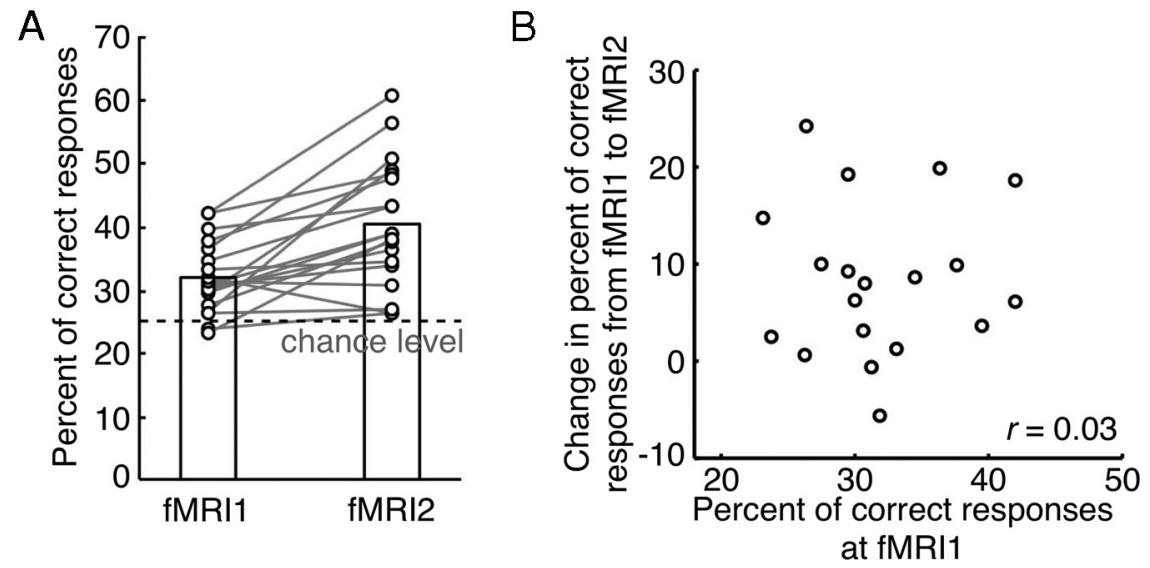

Figure 3. Capability to quickly generate the best next-move developed through the long-term training. $\boldsymbol{A}$, The performance (percent of correct responses) of the subjects in the fMRI experiments (fMRI1 and fMRI2). Gray lines connect data points of the same subject. $\boldsymbol{B}$, The performance at fMRI1 was uncorrelated with the change in performance from fMRI1 to fMRI2 $(r=0.03, p=0.45)$.
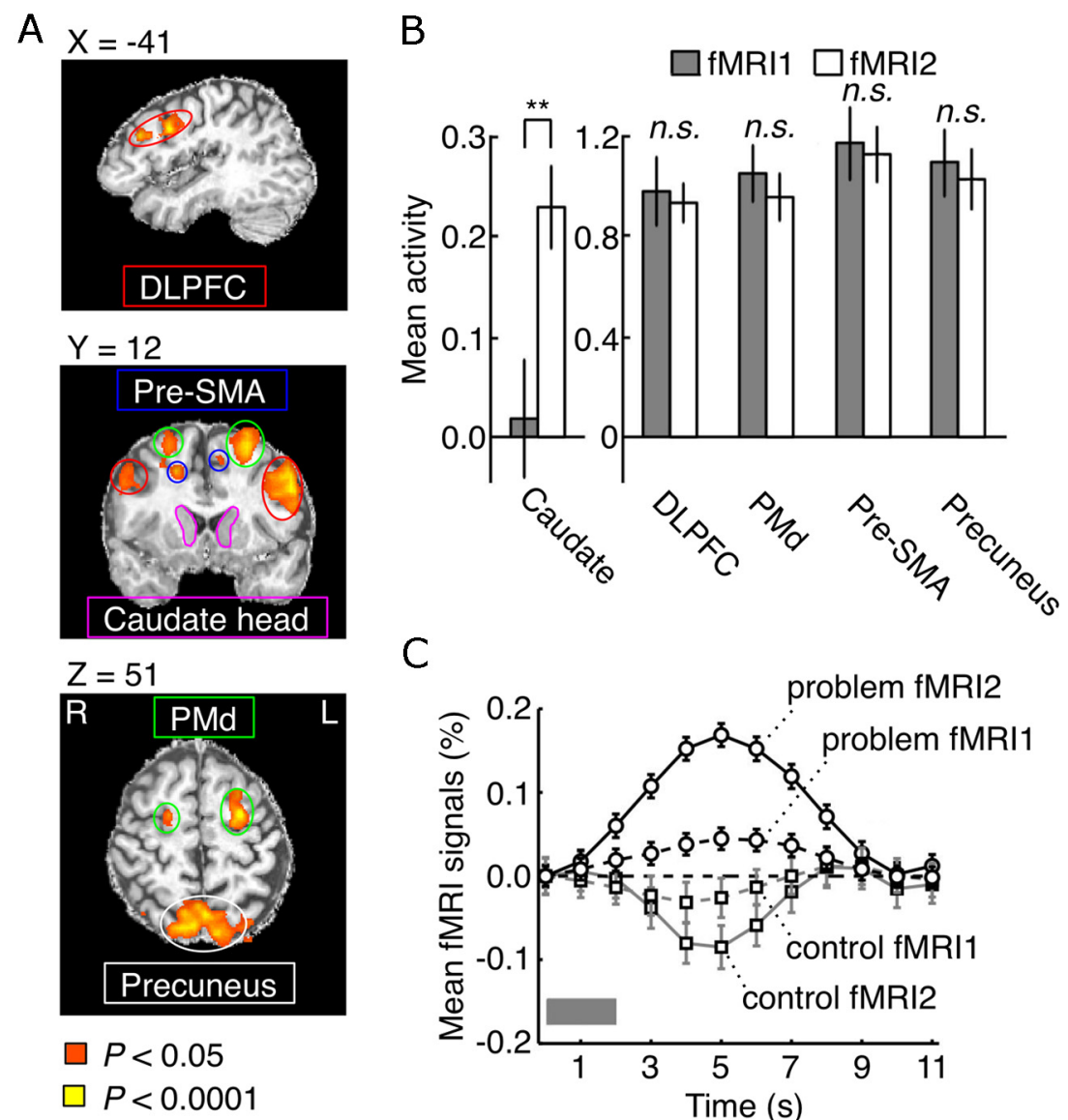

C

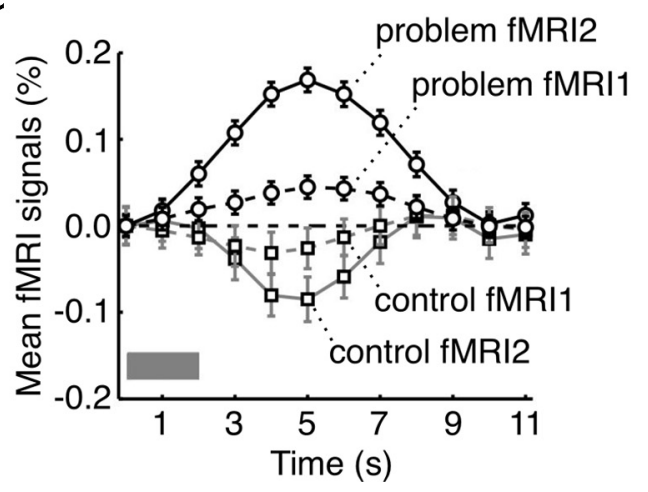

Figure 4. Caudate head activity, but not cortical activities, tracked development of the capability to quickly generate the best next-move. $A$, The activation during the quick generation task in comparison with the activity during the sensorymotor control task ( $p<0.05$, FDR corrected for the whole brain). The foci activated at both FMRI1 and fMRI2 are shown. Significant activations were found in multiple cortical areas, including dorsolateral prefrontal cortex [DLPFC, Talairach coordinates (X/Y/Z): left (L): - 44/4/33; right (R): 42/6/32; circumscribed by red lines], dorsal premotor cortex (PMd, left: -23/1/56; right: 22/-2/56; green lines), pre-supplementary motor area (Pre-SMA, left: $-4 / 14 / 48$; right: 8/15/44; blue lines), and posterior precuneus (Precuneus, left: $-11 /-69 / 53$; right: 12/-72/55; white lines). They were the same as those found in our previous study (Wan et al., 2011) by contrasting the quick generation task with the "Gold"-piece detection task. Although the activation in caudate head at fMRI2 was stronger than that at fMRI1, both did not survive for the multiple-comparison correction. $\boldsymbol{B}$, The activity in the anatomically identified caudate head at $\mathrm{fMRI2}$ (indicated by pink lines in $\boldsymbol{A}$ ) was significantly larger than that at fMRI1 $(p<0.01)$, but activities in cortical areas were not significantly different between fMRI1 and fMRI2 $(p>0.10)$. C, The mean fMRI signal time courses in the caudate head. Error bars in $\boldsymbol{B}$ and $C$ indicate SEM across subjects. the idea of the best next-move. These regressors were then convolved with a canonical, two-gamma hemodynamic response function. Activations associated with the sensory-motor control task were analyzed in the same way. The regression beta coefficients were computed for each individual subject, which were then subjected to a group random-effect analysis of variance. For the activations in the whole brain, the false discovery rate (FDR) was used for multiple-comparison correction $(p<$ 0.05).

For the quantification of activation, the anatomical region of interest (ROI) for the head of caudate nucleus was identified on anatomical images acquired for each subject. The dorsolateral prefrontal cortex (DLPFC), dorsal premotor cortex (PMd), pre-supplementary motor area (pre-SMA), and precuneus ROIs were defined by the voxels activated during the quick generation of the best next-move in comparison with their activity during the sensory-motor control task using the conjunction analysis across fMRI1 and fMRI2 $(p<$ 0.05 , FDR corrected).

The correlations of activation variances were calculated between the ROIs of caudate head and cortical areas, which were defined as described above. The response at each time point in each trial for each ROI in each subject was calculated by averaging fMRI signals over voxels in the ROI. The values were weighted by the two-gamma hemodynamic response function time-locked to the phase of pattern presentation. For each time point in a trial, the mean value averaged across trials was subtracted from the values of individual trials. Then, the correlation was calculated between the values of individual time points from respective pairs of ROIs in each subject. To statistically compare the strength of correlation between different conditions, the correlation coefficients were converted to a normal distribution by Fisher's transformation (Jekins and Watts, 1968), and then the differences between conditions were evaluated by $Z$ test. Because individual time points of fMRI signals in each trial were not independent, the degrees of freedom were modified by the Bartlett correction factor (Jekins and Watts, 1968).

Anatomical analyses. To obtain spatially homogenous high-resolution 3D anatomical images, original $\mathrm{T}_{1} 3 \mathrm{D}$ anatomical images were divided voxel-by-voxel by corresponding $\mathrm{T}_{2}^{*}$ 3D anatomical image (Van de Moortele et al., 2009). Resulting $\left(\mathrm{T}_{1} / \mathrm{T}_{2}^{*}\right) 3 \mathrm{D}$ anatomical images were used for voxel-based morphometry (VBM) and volumetric analyses.

The VBM analysis was performed using the DARTEL module (Ashburner, 2007) included in SPM8 (http://www.fil.ion.ucl.ac.uk/spm). First, all images were segmented into the gray matter (GM), white matter (WM), and cerebrospinal fluid in the original image space. The GM and WM segments from all subjects were then fed together into the DARTEL (Diffeomorphic Anatomical Registration through Exponentiated Lie) module to create a common refined template. Through this common tem- 
plate, original GM segments were warped into a normalized (MNI) space. Normalized images were then spatially smoothed with a Gaussian kernel of $8 \mathrm{~mm}$ (full width at half-maximum). Processed GM images were finally regressed with the percentages of correct responses in fMRI1 and fMRI2, respectively, by a multiple regression model.

The volumetric analysis was conducted using a Bayesian model-based automatic segmentation of subcortical structure tool [FIRST (Patenaude et al., 2011)]. The bilateral caudate nucleus, putamen, globus pallidus, nucleus accumbens, thalamus, amygdala, and hippocampus were labeled and segmented. Segmented subcortical structures for each subject were visually inspected and corrected manually if necessary. The volumetric size of each subcortical structure was adjusted with the subject's intracranial volumes (ICVs), namely, the adjusted volume $=$ raw volume $-b \times(\mathrm{ICV}-$ mean ICV $)$, where ICV was measured from the subject, mean ICV was the averaged ICV across all subjects, and $b$ is the slope of the regressed ICV-subject curve. The adjusted volumetric sizes of subcortical structures were then used to regress with the percentages of correct responses in fMRI1 and fMRI2, as well as the difference in the percentages of correct responses between the two experiments.

IQ and impulsivity tests. The subjects' general intelligence quotient (IQ) was tested by Raven's progressive matrices (http://iqtest.dk/main. swf). Impulsivity was assessed by the Barratt Impulsiveness Scale with 30 items (BIS-11, Patton et al., 1995).

Memory test. Most of the best-next-move-generation problems (160 of 180 ) were repeatedly used in fMRI1 and fMRI2. Because each problem was presented briefly $(2 s)$ and the subjects played many games in the 3 month interval between fMRI1 and fMRI2, it was unlikely that they recalled the problems and correct answers when the same problems appeared again at fMRI2. Nevertheless, to confirm that it was the case, we conducted a psychological experiment immediately after fMRI2. We presented 20 problems that were used only at fMRI1 but not at fMRI2, together with 20 new problems in a random order, and asked the subject whether he had experienced the problem. The pattern of each problem was presented for $2 \mathrm{~s}$, and the subject replied by pressing a button within $3 \mathrm{~s}$. The sensitivity of discriminations ( $\left.\mathrm{d}^{\prime}\right)$ was calculated for each subject.

\section{Results}

We used a game called mini-shogi, which is a simplified version of shogi with a smaller board ( 5 by 5 , instead of 9 by 9 in the original shogi) and fewer types of pieces (Fig. 1), with the expectation that skill acquisition would be faster in simpler games. We recruited 20 novice subjects who had little knowledge or experience in either shogi or mini-shogi and let them practice minishogi games daily for 15 weeks with a computer program. The subjects were motivated for practice by monetary awards differentially given to them according to their results in a tournament held at the end of the training. The accumulated time of practice ranged from 37 to $107 \mathrm{~h}$.

To assess the capability of intuition in fMRI experiments, we used a quick next-move generation task similar to the one used in our previous study (Wan et al., 2011). The board pattern of a checkmate problem of mini-shogi was presented for $2 \mathrm{~s}$, and the subject selected the best next-move from subsequently presented four alternatives by pressing a button within $3 \mathrm{~s}$ (Fig. 2B). Checkmate problems usually require the player to find a series of moves that reach a checkmate (capturing the opponent's King) even when the opponent makes optimal counter moves. To emphasize the intuitive generation of the best next-move, we used the short presentation time and asked the subjects to report only the first

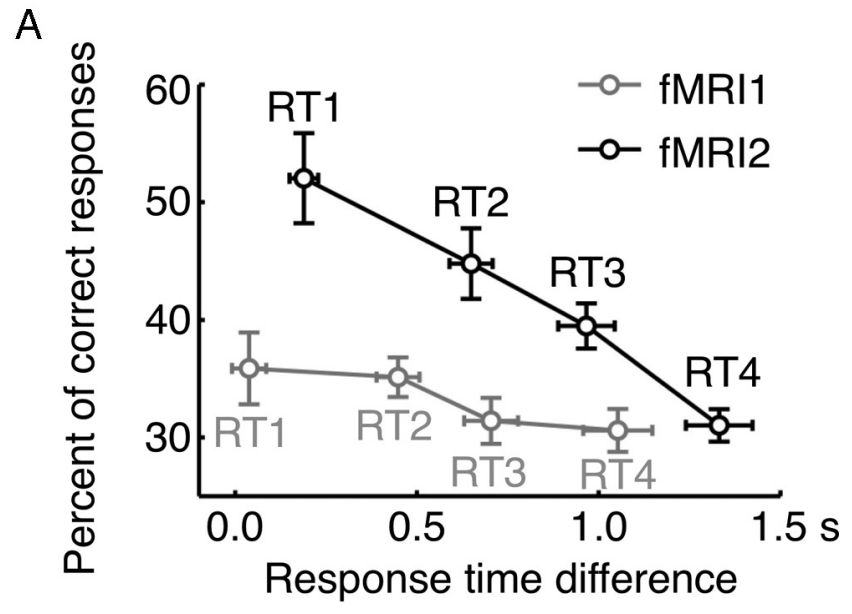

B

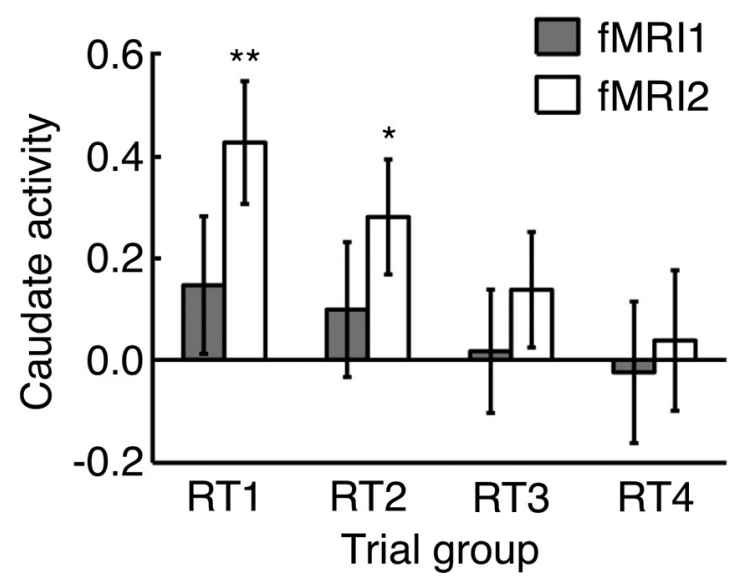

Figure 6. Behavioral performance and caudate head activity were negatively correlated with the response time (RT) at fMRI2. A, The trials of the quick generation task were divided into quarters according to the response time in each subject, and the mean of the averaged percentage of correct responses (the chance level: $25 \%$ ) is plotted against the mean of the averaged response time across groups. They were negatively correlated at fMRI2 (model-II regression, mean regression coefficient $=-0.66, \mathrm{SEM}=0.19, t_{18}=3.49, p=0.001$ ), but not at fMRI1 (model-II regression, mean regression coefficient $=-0.10, \mathrm{SEM}=0.30, t_{18}=0.34, p=$ 0.37 ). The response time difference is the time obtained by subtracting the mean response time in the sensory-motor control trials in each subject from the response time. $\boldsymbol{B}$, The mean caudate head activity in the four groups of trials (RT1-4, RT1 with the shortest response times). The caudate head activity was only significant in the RT1 and RT2 groups at fMRI2. ${ }^{* *} p<0.01$, ${ }^{*} p<0.05$. Error bars indicate SEM across subjects. 

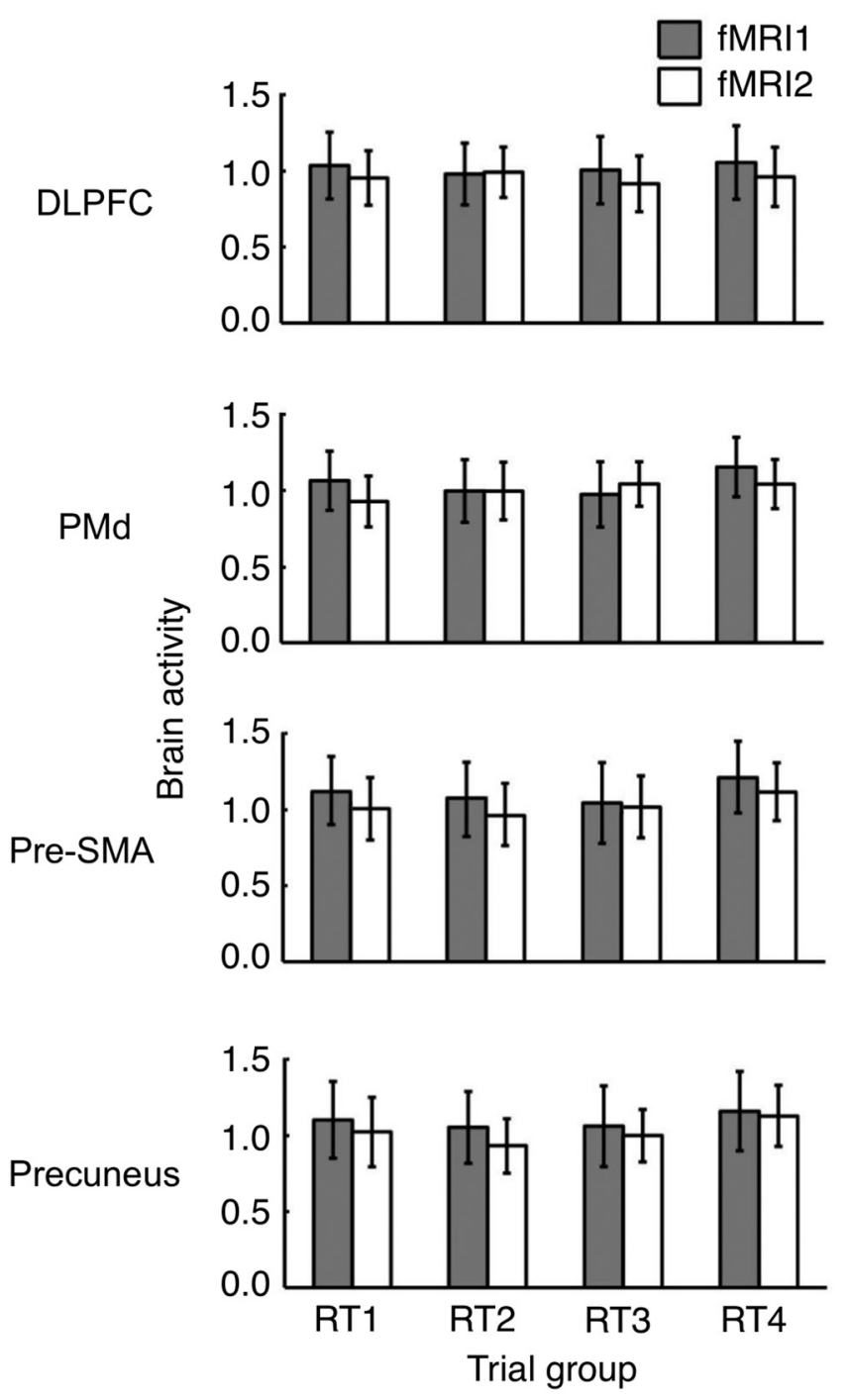

Figure 7. Cortical activities were independent of the subject's response time. The magnitude of the activations in the dorsolateral prefrontal cortex, dorsal premotor cortex, presupplementary motor area, and posterior precuneus at fMRI 1 and fMRI 2 is plotted separately for the four trial groups. Two-way ANOVA with factors of trial group (RT1 4) and history (fMRI1/ fMRI2) showed no main effect of trial group $\left(F_{(3,144)}=1.8, p=0.15\right.$ for $\operatorname{DLPFC}, F_{(3,144)}=1.6$, $p=0.19$ for PMd, $F_{(3,144)}=2.1, p=0.10$ for pre-SMA, $F_{(3,144)}=2.2, p=0.09$ for precuneus) or history $\left(F_{(1,144)}=2.4, p=0.12\right.$ for DLPFC, $F_{(1,144)}=2.1, p=0.15$ for PMd, $F_{(1,144)}=2.3$, $p=0.13$ for pre-SMA, $F_{(1,144)}=2.6, p=0.11$ for precuneus), as well as interaction between the two factors $\left(F_{(3,144)}=1.1, p=0.35\right.$ for DLPFC, $F_{(3,144)}=1.3, p=0.28$ for PMd, $F_{(3,144)}=$ $1.4, p=0.24$ for pre-SMA, $F_{(3,144)}=1.3, p=0.28$ for precuneus). Error bars indicate SEM across subjects.

move. In interviews following the fMRI experiments, the subjects commonly reported that they got the idea of the best next-move without deliberate, explicit thinking. Trials of the quick generation task were randomly intermingled with those of a sensory motor control task in which a board pattern composed only of the opponent's pieces was presented and the subject reported the King's position (Fig. 2B). The ideas of next moves could not occur in these control trials because of the absence of the player's own pieces.

\section{Behavioral results}

The training significantly improved the general skill in playing mini-shogi games in all subjects. The mean percentage of correct responses in the quick generation task, measured in the fMRI
A

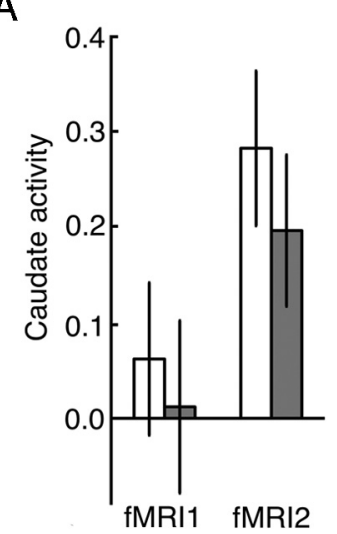

$\mathrm{B}$

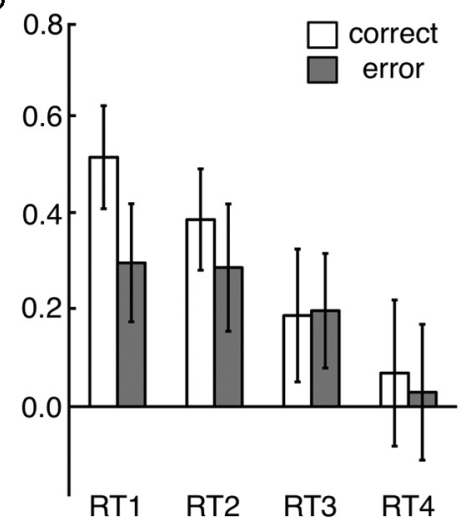

Figure 8. Caudate head activity in correct and error trials. $\boldsymbol{A}$, Shown for correct and error trials at $\mathrm{fMRI} 1$ and $\mathrm{fMRI2}$, respectively. $\boldsymbol{B}$, Shown separately are four trial groups divided according to the response time (RT) in individual subjects at fMRI2. The activity in error trials was not different from that in correct trials as a whole $(p=0.11)$ or in any of the four trial groups ( $p>$ 0.05). Error bars indicate SEM across subjects.

Table 1. Coefficients of correlation between caudate head and cortical activations

\begin{tabular}{|c|c|c|c|c|c|c|c|}
\hline & \multicolumn{3}{|l|}{ fMRI1 } & \multicolumn{3}{|l|}{ fMRI2 } & \multirow{2}{*}{$\begin{array}{l}p \text { value of } \\
\text { fMRI } 1 / f M R^{\text {ffMrerence }}\end{array}$} \\
\hline & $\begin{array}{l}\text { Next-move } \\
\text { generation }\end{array}$ & Control & $\begin{array}{l}p \text { value of } \\
\text { difference }\end{array}$ & $\begin{array}{l}\text { Next-move } \\
\text { generation }\end{array}$ & Control & $\begin{array}{l}p \text { value of } \\
\text { difference }\end{array}$ & \\
\hline DLPFC & $\begin{array}{c}0.17^{b} \\
(0.06)^{c}\end{array}$ & $\begin{array}{c}0.15 \\
(0.07)\end{array}$ & 0.37 & $\begin{array}{c}0.29 \\
(0.05)\end{array}$ & $\begin{array}{c}0.17 \\
(0.06)\end{array}$ & 0.016 & 0.019 \\
\hline Pre-SMA & $\begin{array}{c}0.18 \\
(0.06)\end{array}$ & $\begin{array}{c}0.16 \\
(0.07)\end{array}$ & 0.42 & $\begin{array}{c}0.30 \\
(0.05)\end{array}$ & $\begin{array}{l}0.17 \\
(0.07)\end{array}$ & 0.014 & 0.016 \\
\hline PMd & $\begin{array}{c}0.17 \\
(0.06)\end{array}$ & $\begin{array}{c}0.16 \\
(0.07)\end{array}$ & 0.44 & $\begin{array}{c}0.31 \\
(0.05)\end{array}$ & $\begin{array}{c}0.17 \\
(0.06)\end{array}$ & 0.006 & 0.007 \\
\hline Precuneus & $\begin{array}{c}0.17 \\
(0.06)\end{array}$ & $\begin{array}{c}0.17 \\
(0.07)\end{array}$ & 0.50 & $\begin{array}{c}0.30 \\
(0.05)\end{array}$ & $\begin{array}{c}0.17 \\
(0.07)\end{array}$ & 0.008 & 0.009 \\
\hline
\end{tabular}

${ }^{a}$ Difference between the coefficients of correlation during the next-move generation task at fMRI 1 and fMRI2. ${ }^{b}$ Mean of coefficients.

'SEM of coefficients across subjects.

experiments, increased considerably from fMRI1 to fMRI2 (31\% to $40 \%, p=0.002$, paired $t$ test, Fig. $3 A$ ). This increase may be better appreciated by looking at the difference of the mean from the chance level (25\%): the difference was $6 \%$ at fMRI 1 and $15 \%$ at $\mathrm{PMRI}$, respectively. The behavioral data also suggest that there were at least two phases in the development of capability to quickly generate the best next-move. The change in the percentage of subject's correct responses from fMRI1 to fMRI2 was uncorrelated with the percentage of correct responses at fMRI1 $(r=$ $0.03, p=0.45$, Fig. $3 B$ ), although it was highly correlated with the percentage of correct responses at fMRI2 $(r=0.82, p=8.6 \times$ $\left.10^{-6}\right)$. This independence between the initial phase (the first 2 weeks, till fMRI1) and the late phase (about 3 months from fMRI1 to fMRI2) of learning indicates that the subjects who acquired the capability to quickly generate the best next-move in the first 2 weeks did not necessarily develop it further over the remaining weeks of the training.

The diversity of subjects' performance at fMRI1 or performance changes from fMRI1 to fMRI2 cannot be explained by the learning time, general IQ, or impulsivity, because none of these factors was correlated with the subject's performance at fMRI1 (learning time: $r=0.16, p=0.26$; IQ: $r=0.20, p=0.21$; impulsivity: $r=0.24, p=0.16)$ or the performance change from fMRI1 to fMRI2 (learning time: $r=0.12, p=0.31$; IQ: $r=0.14, p=$ 0.28 ; impulsivity: $r=0.17, p=0.24$ ). Although most of the best-next-move-generation problems (160 of180) were repeat- 
edly used in fMRI1 and fMRI2, the memory tests conducted immediately after fMRI2 on the 20 checkmate problems used at fMRI1 (intermingled with 20 new problems) showed that the $\mathrm{d}^{\prime}$ between the experienced and new problems was small $(-0.022 \pm 0.375$, mean $\pm \mathrm{SD}$, one-tailed $t$ test, $p=0.24)$. Furthermore, there was no correlation between the $\mathrm{d}^{\prime}$ and the percentage of correct responses at fMRI2 $(r=$ $0.09, p=0.36$ ). These results indicate that the subjects did not remember the problems experienced at fMRI1 when they saw them again at fMRI2.

\section{fMRI results}

Brain activities locked to the game problem presentation and subsequent response time in the quick generation task were compared with activities locked to the corresponding periods in the sensorymotor control task. Activations at multiple cortical areas, including the dorsolateral prefrontal cortex, dorsal premotor cortex, pre-supplementary motor area, and posterior precuneus, were observed at both fMRI1 and fMRI2 (Fig. $4 A$ ). However, the magnitudes of these cortical activations did not change from fMRI1 to fMRI2 (Fig. $4 B$, right), nor did they correlate with the percentage of correct responses during fMRI experiments $(p>0.28)$.

In contrast, activity in the anatomically identified caudate head showed a significant increase from fMRI1 to fMRI2 (Fig. $4 B$, left, and Fig. 4C). Caudate head activity in individual subjects measured at fMRI2 was correlated with the change in the percentage of correct responses from fMRI1 to fMRI2 $(r=0.46, p=0.024$, Fig. $5 A)$, whereas there was no correlation between caudate head activity at fMRI1 and the percentage of correct responses at fMRI1 $(r=0.12, p=0.31$, Fig. $5 B)$. Thus, a close relationship between the performance in the quick generation of the best next-move and caudate head activity was not present after the initial training (until fMRI1) but developed during the training between fMRI1 and fMRI2.

The subjects' behavioral performance and caudate head activations at fMRI2 exhibited a similar dependence on response time to that seen in high-level amateur players in our previous study with original shogi (Wan et al., 2011). When the task trials were divided into quarters for each subject according to his response time, the average percentage of correct responses was higher (Fig. 6A) and caudate head activity was stronger (Fig. 6B) in the trial quarter with shorter response times. Such a pattern was not observed, either in behavioral performance or in caudate head activity, at fMRI1. In comparison, such a dependence on the response time was not seen in cortical activations at either fMRI1 or fMRI2 (Fig. 7).

In addition, we confirmed that the caudate activation associated with the quick generation of the best next-move at fMRI2 was independent of the correctness of responses. The caudate activities were significant even in error trials (Fig. $8 A, p=0.03$ ), and there were no significant differences in the magnitude of
A

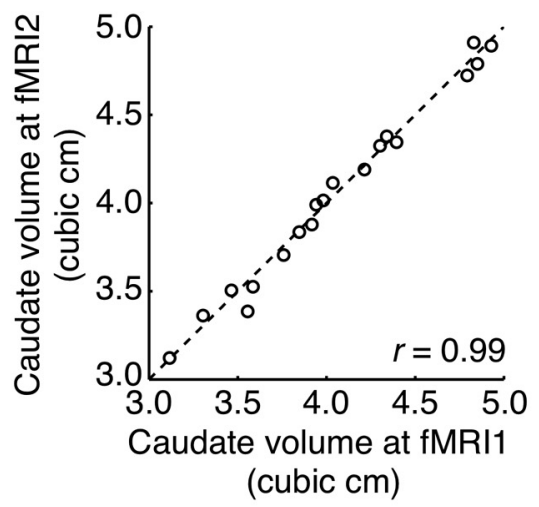

C

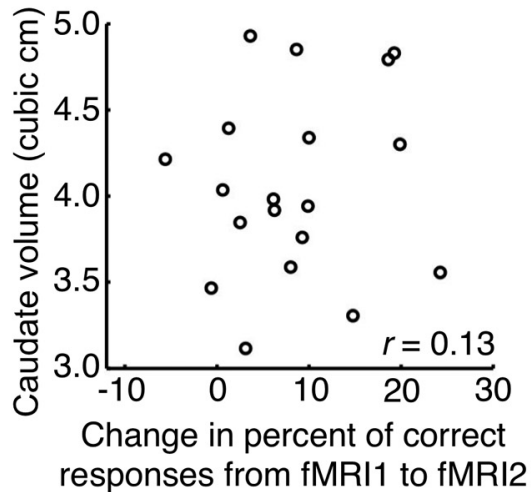

caudate activities between correct and error trials (Fig. $8 A, p=$ 0.11 across all trials, and Fig. $8 B, p>0.05$ for the quarter groups of trials binned according to the response time).

Finally, the correlations between the caudate head and cortical activations during the quick generation of the best next-move were significantly larger than those during the sensory-motor control task at fMRI2, whereas there was no such difference at fMRI1 (Table 1). Overall, the correlations during the quick generation of the best next-move were significantly larger at fMRI2 than those at fMRI1 (Table 1).

\section{Anatomical results}

Several studies have documented that expertise or better performance in specific domains is associated with the larger size of particular brain areas/structures (Maguire et al., 2000, Erickson et al., 2010). A change in the volume of a particular cortical area after training of a particular motor skill has also been reported (Draganski et al., 2004). We thus examined whether there was a correlation between the development of capability to quickly generate the best next-move and the volume and/or volume change of certain anatomical brain structures, including the caudate nucleus. We applied both the voxel-based morphometry (VBM) analysis to the whole brain and volumetric analysis to subcortical structures (see Materials and Methods) and found that, in either analysis, there was no apparent structural change from fMRI1 to fMRI2, including the volume of the caudate nu- 
A
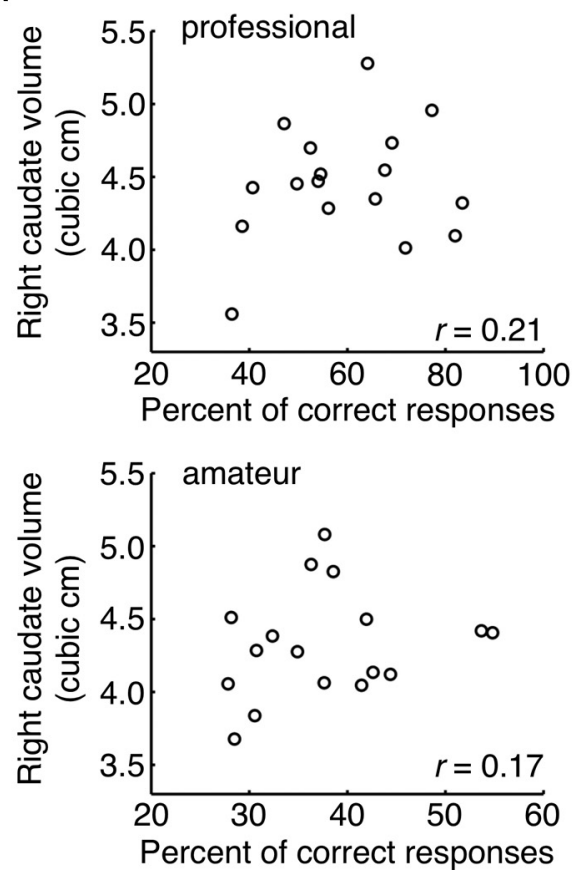

Figure 10. Right caudate volume and subject's performance in the task to quickly generate the bestnext-move in original shogi (for task details, see Wan et al., 2011). $\boldsymbol{A}$, The right caudate volume was uncorrelated with the subject's performance in the quick generation task of original shogi, either for professional players $(n=17, r=0.21, p=0.20)$ or high-level amateur players $(n=17, r=0.17, p=0.26) \cdot \boldsymbol{B}$, The right caudate volume was not significantly different between professional and amateur groups $\left(t_{16}=1.42, p=0.09\right)$.

\section{Discussion}

In summary, by using mini-shogi, a simplified version of shogi, we succeeded in training novices within several months to develop the near-expert capability to quickly generate the best next-move to a given board pattern. Task-specific activation in the caudate head and its correlation with performance were absent in the early phase of training but appeared at the end of training. These results are indicative of a tight coupling between the recruitment of the caudate head and the development of quick generation capability through extensive training. Although there was no change in cortical activation from the early phase to the end of training, a task-specific correlation appeared between the caudate and cortical activities after the training. The emergent coherence suggests that cortical information processing may have also changed as a result of training so that a stronger interaction is forged between cortical areas and the caudate head.

Novices play a board game by consciously generating a branching series of self-moves and opponent's countermoves, evaluating them by their consequences, and selecting the best next-move that would result in the best consequence

cleus $(p=0.43$ for the right caudate nucleus and $p>0.20$ for all other subcortical areas by paired $t$ test; Fig. $9 A$ ). The small numerical differences in the measured size of the caudate nucleus between the two experiments were not correlated with subjects' performance changes from fMRI1 to fMRI2 ( $r=$ $0.09, p<0.36$ ).

The volume of a subject's caudate nucleus on the right was positively correlated with the subject's performance in the quick generation task at fMRI1 $(r=0.50, p=0.014$, uncorrected, Fig. $9 B$ ) and at fMRI2 ( $r=0.42, p=0.036$, uncorrected), but not with the change in performance from fMRI1 to fMRI2 $(r=0.13, p=$ 0.30 , Fig. $9 C$ ). No other subcortical structures, including the left caudate nucleus, showed a significant correlation in volume with an individual's performance at fMRI1, fMRI2, or the change in performance from fMRI1 to fMRI2. The VBM analysis did not reveal any brain region that showed a significant correlation with the performance or the performance change. In brief, it appears that the volume of the right caudate nucleus constrained only the initial phase of development of the capability to quickly generate the best nextmove, but not the later, main phase of development.

The dissociation of development of quick generation capability in the late phase from the volume of the caudate nucleus suggests that the volume of the caudate nucleus per se would not constrain the level of capability to quickly generate the best nextmove achieved after years of extensive training. In reexamining the data from our previous study, as a matter of fact, we found no correlation in either professional or high-level amateur shogi players between the volume of the caudate nucleus and performance in the quick best next-move-generation task with problems from original shogi (Fig. 10A). There was also no significant difference in the volume of the caudate nucleus between professional and high-level amateur players (Fig. 10B). (conscious search). As the experience accumulates, the generation of ideas of the best next-moves becomes automatic and quick. This automatic, quick process is largely unconscious in that the player cannot explain how the idea is generated and why it is the best. Psychological studies of chess and shogi have shown that the capability of this automatic, quick generation, which has been termed "intuition," is essential for the superior capability of expert players (De Groot, 1965, 1986; Gobet and Chassy, 2009). In real games, experts do depend on conscious search in their selections of next moves, but their search procedures, supported by high intuitive capability, are much more effective.

A simple contrast of the task to generate the best next-move with the sensory-motor control task would not have isolated the cognitive component to intuitively generate the best next-move. However, the short presentation time $(2 \mathrm{~s})$ and brief response window ( $3 \mathrm{~s}$ ) were critical in minimizing the contribution of conscious search. These durations were determined in preliminary experiments with a separate group of subjects who had gone through similar amounts of training to those of the subjects in the main experiment. When we gradually increased the presentation time and response window from $1 \mathrm{~s}$ and $2 \mathrm{~s}$, respectively, the subjects' performance became significantly higher than chance at these values. The actual response time, after subtracting the mean response time for the sensory-motor task in each subject, was shorter than $1.0 \mathrm{~s}$ in $84 \%$ of the trials (Fig. $6 \mathrm{~A}$ ). In interviews conducted after the fMRI experiments, the subjects commonly reported that they could not make a conscious search. Moreover, the negative correlation between the performance and response time (the mean percentage of correct responses was higher in trials with shorter response times) after extensive training (at fMRI2) supports our contention that the generation of the best 
next-move depended largely on intuition, as a negative correlation is inconsistent with the essential contribution of conscious search to the response generation. Had the response depended on conscious search, there should have been a time-accuracy tradeoff, thus resulting in a positive correlation between the performance and response time. Based on these facts, we conclude that the performance in the quick generation task was largely determined by the level of the capability to intuitively generate the best next-move, and that the performance improvement in the quick generation task through extensive training represented the development of subjects' intuition in the domain of mini-shogi.

Our previous study included a task condition that tapped conscious search (Wan et al., 2011). In a separate session, the checkmate problems to which the subject had responded erroneously in the preceding sessions with the $1 \mathrm{~s}$ condition were presented again, and the subject was allowed $8 \mathrm{~s}$ to generate a response. We found that $8 \mathrm{~s}$ were sufficiently long for both professional and high-rank amateur players to make conscious search. Importantly, the caudate nucleus was not activated in this $8 \mathrm{~s}$ condition. Thus, the caudate activations were specific to the $1 \mathrm{~s}$ condition, which was designed to tap the intuitive generation. On the other hand, cortical activations were common to $1 \mathrm{~s}$ and $8 \mathrm{~s}$ conditions. In addition, the magnitude of caudate activations was significantly stronger in professional players than those in high-rank amateurs, which is in line with the results from psychological studies on board game experts, showing that world-top-class players have developed much higher intuition capability than high-level amateurs (De Groot, 1965, 1986). Based on these findings, we had previously concluded that the caudate activation is specifically associated with quick generation of the best nextmove. We had also suggested that the association might have developed as a result of extensive training. However, there had been no direct evidence supporting this hypothesis. In the current study we have extended our previous conclusion by demonstrating that the caudate activation indeed develops through long-term extensive training, in parallel to the development of the subject's capability to quickly generate the best next-move to a given board pattern.

The striatum is also activated in response to rewards (Schultz, 2000; Delgado, 2007). Although there was no explicit feedback to the subjects about the correctness of responses in our task, some implicit rewards might have occurred when the subjects felt confident in their responses. However, at fMRI2, the caudate head was significantly activated even in error trials, and there was no significant difference in the magnitude of caudate activities between correct and error trials (Fig. 8). The confidence judgment made after response in our previous study had revealed a close relation between the subject's confidence and the correctness of responses: amateur players reported that they were confident in $78 \%$ of correct trials but were not confident in $61 \%$ of error trials. Taken together, it is unlikely that implicit rewards from the sense of feeling successful can account for the pattern of caudate activations observed in the present study.

Although the current study doesn't explicitly demonstrate exactly what information processing takes places in the caudate head during the intuitive generation of the best next-move, some hints can be found in the rich literature on structures and functions of the basal ganglia. The basal ganglia are connected with cortical areas via cortico-striato-thalamic loops and contain a unique circuit for "inhibition of inhibition." The classical concept of parallel loops (Alexander et al., 1986) has been modified by findings of considerable convergence in cortico-striate projections (Graybiel, 1998; Haber et al., 2006). The dorsal caudate head receives extensive projections from the parietal and dorsal anterior cingulate areas in addition to those from the dorsolateral prefrontal area (Selemon and Goldman-Rakic, 1985; Yeterian and Pandya, 1993, 1995; Haber et al., 2006). The converging cortico-striatal projections may help gather relevant information from cortical areas. Then, the parallel combination of inhibition-of-inhibition circuit (direct pathway) and excitation-of-inhibition via subthalamic nucleus circuit (hyperdirect pathway) aids the selection of one response plan from many options (Berns and Sejnowski, 1998). Furthermore, the rich innervation with dopamine neurons provides the basal ganglia opportunities of efficient reinforcement learning (Houk et al., 1995; Montague et al., 1996; Schultz et al., 1997). The selection of response plan may be mediated by the comparison of values, or outcome contingencies, of possible options (Samejima et al., 2005). By organizing these loops for the vast knowledge and experience about mini-shogi games through extensive training, the processes to generate the best next-move become fast and automatic or, namely, intuitive (Graybiel, 1998; Lieberman, 2000; Wan et al., 2011).

The volume of the caudate nucleus did not constrain the intuition development in the main part of training in the present study, although it was correlated with the development in the initial 2 weeks of training. Also, there was no systematic difference in the caudate volume between professional and high-level amateur shogi players. In amateurs, the circuits, including the caudate head, may be also used for different purposes including various types of decision-making in daily life. Thus, the overall degree to which the circuits are used may be not significantly different between shogi professionals and amateurs.

The training period used in the present study ( 15 weeks) was shorter than the typical period of domain-specific training that has been assumed to be necessary to produce expertise at the professional level (10 years) (Simon and Chase, 1973; Ericsson and Lehmann, 1996). The simplicity of the mini-shogi game enabled faster development of intuition in the present study. However, the caudate activation found in the current study was more similar to that of high-level amateur shogi players rather than that of professional shogi players in our previous study in that it depended on the response time (Wan et al., 2011). The same kind of dependency on the response time was found in high-level amateurs, but not in professionals, in our previous study. The caudate head activation was always strong in professionals. By continuing the training for a longer period (e.g., 10 years) and also making the daily training more rigorous, the capability of fully automatic processing by circuitries, including the caudate head, may reach a professional level.

\section{References}

Alexander GE, DeLong MR, Strick PL (1986) Parallel organization of functionally segregated circuits linking basal ganglia and cortex. Annu Rev Neurosci 9:357-381. CrossRef Medline

Anderson JR (1982) Acquisition of cognitive skill. Psychol Rev 89:369-406. CrossRef

Ashburner J (2007) Fast diffeomorphic image registration algorithm. Neuroimage 38:95-113. CrossRef Medline

Berns GS, Sejnowski TJ (1998) A computational model of how the basal ganglia produce sequences. J Cogn Neurosci 10:108-121. CrossRef Medline

Dahlin E, Neely AS, Larsson A, Bäckman L, Nyberg L (2008) Transfer of learning after updating training mediated by the striatum. Science 320: 1510-1512. CrossRef Medline

De Groot AD (1965) Thought and choice in chess (translated from the Dutch original, 1946). The Hague: Mouton.

De Groot AD (1986) Intuition in chess. ICCA J 9:67-75.

Delgado MR (2007) Reward-related responses in the human striatum. Ann NY Acad Sci 1104:70-88. CrossRef Medline 
Draganski B, Gaser C, Busch V, Schuierer G, Bogdahn U, May A (2004) Neuroplasticity: changes in grey matter induced by training. Nature 427 : 311-312. CrossRef Medline

Dux PE, Tombu MN, Harrison S, Rogers BP, Tong F, Marois R (2009) Training improves multitasking performance by increasing the speed of information processing in human prefrontal cortex. Neuron 63:127-138. CrossRef Medline

Erickson KI, Colcombe SJ, Wadhwa R, Bherer L, Peterson MS, Scalf PE, Kim JS, Alvarado M, Kramer AF (2007) Training-induced functional activation changes in dual-task processing: an fMRI study. Cereb Cortex 17: 192-204. CrossRef Medline

Erickson KI, Boot WR, Basak C, Neider MB, Prakash RS, Voss MW, Graybiel AM, Simons DJ, Fabiani M, Gratton G, Kramer AF (2010) Striatal volume predicts level of video game skill acquisition. Cereb Cortex 20:25222530. CrossRef Medline

Ericsson KA, Lehmann AC (1996) Expert and exceptional performance: evidence of maximal adaptation to task constraints. Annu Rev Psychol 47: 273-305. CrossRef Medline

Fitts PM, Posner MI (167) Human performance. Monterey, CA: Brooks/ Cole.

Gobet F, Chassy P (2009) Expertise and intuition: a tale of three theories. Minds Mach 19:151-180. CrossRef

Graybiel AM (1998) The basal ganglia and chunking of action repertories. Neurobiol Learn Mem 70:119-136. CrossRef Medline

Haber SN, Kim KS, Mailly P, Calzavara R (2006) Reward-related cortical inputs define a large striatal region in primates that interface with associative cortical connections, providing a substrate for incentive-based learning. J Neurosci 26:8368-8376. CrossRef Medline

Houk JC, Adams JL, Barto AG (1995) A model of how the basal ganglia generate and use neural signals that predict reinforcement. In: Models of information processing in the basal ganglia (Houk JC, Davis JL, Beiser DG, eds), pp 249-270. Cambridge, MA: MIT.

Jekins GM, Watts DG (1968) Spectral analysis and its applications. San Francisco: Holden-Day.

Kellman P, Epstein FH, McVeigh ER (2001) Adaptive sensitivity encoding incorporating temporal filtering (TSENSE). Magn Reson Med 45: 846-852. CrossRef Medline

Kelly AM, Garavan H (2005) Human functional neuroimaging of brain changes associated with practice. Cereb Cortex 15:1089-1102. CrossRef Medline

Klein GA (2003) Intuition at work. New York: Doubleday.

Lieberman MD (2000) Intuition: a social cognitive neuroscience approach. Psychol Bull 126:109-137. CrossRef Medline

Maguire EA, Gadian DG, Johnsrude IS, Good CD, Ashburner J, Frackowiak RS, Frith CD (2000) Navigation-related structural change in the hip- pocampi of taxi drivers. Proc Natl Acad Sci U S A 97:4398-4403. CrossRef Medline

Montague PR, Dayan P, Sejnowski TJ (1996) A framework for mesencephalic dopamine systems based on predictive Hebbian learning. J Neurosci 16:1936-1947. Medline

Olesen PJ, Westerberg H, Klingberg T (2004) Increased prefrontal and parietal activity after training of working memory. Nat Neurosci 7:75-79. CrossRef Medline

Patenaude B, Smith SM, Kennedy DN, Jenkinson M (2011) Bayesian model of shape and appearance for subcortical brain segmentation. Neuroimage 56:907-922. CrossRef Medline

Patton JH, Stanford MS, Barratt ES (1995) Factor structure of the barratt impulsiveness scale. J Clin Psychol 51:768-774. CrossRef Medline

Poldrack RA (2000) Imaging brain plasticity: conceptual and methodological issues-a theoretical review. Neuroimage 12:1-13. CrossRef Medline

Poldrack RA, Gabrieli JD (2001) Characterizing the neural mechanisms of skill learning and repetition priming evidence from mirror reading. Brain 124:67-82. CrossRef Medline

Samejima K, Ueda Y, Doya K, Kimura M (2005) Representation of actionspecific reward values in the striatum. Science 310:1337-1340. CrossRef Medline

Schultz W (2000) Multiple reward signals in the brain. Nat Rev Neurosci 1:199-207. CrossRef Medline

Schultz W, Dayan P, Montague PR (1997) A neural substrate of prediction and reward. Science 275:1593-1599. CrossRef Medline

Selemon LD, Goldman-Rakic PS (1985) Longitudinal topography and interdigitation of corticostriatal projections in the rhesus monkey. J Neurosci 5:776-794. Medline

Simon HA, Chase WG (1973) Skill in chess. Am Sci 61:393-403.

Talairach J, Tournoux P (1988) Co-planar stereotaxic atlas of the human brain. New York: Thieme.

Van de Moortele PF, Auerbach EJ, Olman C, Yacoub E, Uğurbil K, Moeller S (2009) $\mathrm{T}_{1}$ weighted brain images at 7 Tesla unbiased for proton density, $\mathrm{T}_{2}^{*}$ contrast and $\mathrm{RF}$ coil receive $\mathrm{B}_{1}$ sensitivity with simultaneous vessel visualization. Neuroimage 46:432-446. CrossRef Medline

VanLehn K (1996) Cognitive skill acquisition. Annu Rev Psychol 47:513539. CrossRef Medline

Wan X, Nakatani H, Ueno K, Asamizuya T, Cheng K, Tanaka K (2011) The neural basis of intuitive best next-move generation in board game experts. Science 331:341-346. CrossRef Medline

Yeterian EH, Pandya DN (1993) Striatal connections of the parietal association cortices in rhesus monkeys. J Comp Neurol 332:175-197. CrossRef Medline

Yeterian EH, Pandya DN (1995) Corticostriatal connections of extrastriate visual areas in rhesus monkeys. J Comp Neurol 352:436-457. CrossRef Medline 